\title{
The potential of natural regeneration of rocky outcrop vegetation on rupestrian field soils in "Serra do Cipó", Brazil
}

\author{
BRANCA MARIA OPAZO MEDINA ${ }^{1,2}$ and GERALDO WILSON FERNANDES ${ }^{1}$
}

(received: October 14, 2004; accepted: September 11, 2007)

\begin{abstract}
The potential of natural regeneration of rocky outcrop vegetation on rupestrian field soils in "Serra do Cipó", Brazil). An area's innate potential to regenerate represents a crucial factor for its conservation and management. The seed rain and seed bank are important agents in the regeneration process. Seed banks are particularly important in communities where there is a high proportion of obligate seeders. Rocky outcrops are habitats where most part of the plant species depends on their seeds to reproduce and maintain viable populations. Therefore, seed banks ought to be important in this vegetation physiognomy. We test the hypotheses that the seed bank of the rocky outcrops found in the rupestrian fields of "Serra do Cipó", Brazil, is richer in species and denser than those formed on different vegetation physiognomies neighboring the outcrops. We then compared species abundance, species richness and composition in the rocky outcrops' seed banks with those of sandy and peaty bogs, forests, gallery forests, and "cerrados". Furthermore, we report on the natural regeneration potential of these soils by assessing a greenhouse study on seedling emergence. Soil samples were collected from 0 to 5 and 5 to $10 \mathrm{~cm}$ of depth. Rocky outcrops had the poorest in species and less dense seed bank and showed segregation in species composition. Emergence was greater in the most superficial layer. However, soils on rocky outcrops showed the greatest proportion of endemic threatened species in their seed banks, demonstrating their importance for biodiversity conservation of the "Serra do Cipó" rupestrian fields.
\end{abstract}

Key words - natural regeneration, rocky outcrops, seed bank, seedling emergence, "Serra do Cipó"

RESUMO - (Potencial regenerativo natural da vegetação rupestre sobre solos de afloramentos rochosos na Serra do Cipó, Brasil). O potencial nato de uma área regenerar após um distúrbio é um fator crucial para sua conservação e manejo. A chuva e o banco de sementes são agentes importantes dessa regeneração. Bancos de sementes são particularmente importantes em comunidades onde a proporção de espécies com reprodução estritamente por sementes é alta. Nesses casos, o banco de sementes é a principal via de regeneração. Afloramentos rochosos são ambientes onde a maior parte das espécies dependem de sementes para reproduzir e manter populações viáveis. Assim, testamos a hipótese de que os afloramentos rochosos da Serra do Cipó teriam solos com bancos de sementes mais ricos e densos do que as outras fisionomias vegetacionais adjacentes. Comparamos então a abundância, riqueza e composição de espécies dos bancos de sementes dos solos dos afloramentos rochosos com os dos brejos arenosos e turfosos, capões de mata, matas de galeria e cerrados. Avaliamos a regeneração natural através da emergência de plântulas. O solo foi coletado entre 0 e $5 \mathrm{~cm}$ e entre 5 e $10 \mathrm{~cm}$ de profundidade. Os afloramentos rochosos apresentaram os bancos menos densos e mais pobres em espécies, sendo a emergência de plântulas maior nas camadas mais superficiais do solo. Entretanto, a proporção de espécies endêmicas e ameaçadas foi maior, demonstrando que esses solos são importantes para a conservação da biodiversidade dos campos rupestres da Serra do Cipó.

Palavras-chave - afloramentos rochosos, banco de sementes, emergência de plântulas, regeneração natural, Serra do Cipó

\section{Introduction}

An area's innate potential to regenerate after a given disturbance represents a crucial factor for its conservation and management (Parker et al. 1989, van der Valk \& Pederson 1989, Williams-Linera 1993). This regeneration potential is strongly influenced by the seed rain and seed bank. The seed bank is considered the most important component of the regenerative niche (Kirkman \& Sharitz

\footnotetext{
1. Universidade Federal de Minas Gerais, ICB, DBG, Ecologia Evolutiva e Biodiversidade, Caixa Postal 486, 30161-970 Belo Horizonte, MG, Brazil.

2._Corresponding author: branca.medina@gmail.com
}

1994) and it is characterized by a seed aggregation capable of recovering the adults (Baker 1989). Seeds maintain and expand populations through dispersion and survival during unfavorable periods (Vazquez-Yanes \& OrozcoSegovia 1993). Otherwise, seeds need to remain viable to perform these functions, with sufficient germinability and/or, sometimes, dormancy (Louda 1989).

The functioning of the seed bank can be assessed by its structure, through the number of viable seeds (Fenner 1995). Viability can be tested by germination and seedling emergence, while germination is the main process of seed bank depletion (Parker et al. 1989). The relative importance of seed recruitment varies between communities, whereas the size and functional significance 
of the seed bank vary between different physiognomies or vegetation types (Louda 1989, Hopkins et al. 1990, Vazquez-Yanes \& Orozco-Segovia 1993). Hence, at a regional scale, a systemic view of the emergence behavior of the species is most adequate to better understand the process of regeneration (Sousa-Silva et al. 2001), such as to compare the richness and density of the seed bank of different vegetation formations (Garwood 1989, Butler \& Chazdon 1998).

Seed banks are particularly important in communities where there is a high proportion of obligate seeders; in these cases, they provide the most important means of regeneration. In rocky outcrops most plant species depend on their seeds to reproduce and maintain viable populations (Clarke \& Knox 2002, Yates \& Ladd 2004, Clarke et al. 2005). The rupestrian field vegetation of SE-Brazil is a unique and rare formation composed of a mosaic of vegetation types, associated to some physiognomies found primarily in the Espinhaço Range. This vegetation mosaic has been under severe and intense impacts caused primarily by mineral and wood extraction, road construction, fire, cattle grazing, and increasing uncontrolled tourism and human settlement (Giulietti \& Menezes 2000, Viana et al. 2005). Most of these disturbances have led to soil erosion, species extinction, and ultimately species replacement caused by biological invasions. In this scenario, natural regeneration studies are crucial for its conservation and management. Although some efforts have been made to understand germination of this unique flora (Gomes et al. 2001, Ranieri et al. 2003, Garcia \& Diniz 2003, Silveira et al. 2004, Abreu \& Garcia 2005, Oliveira \& Garcia 2005, Silveira et al. 2005, Velten \& Garcia 2005, Garcia et al. 2007), none has yet addressed the natural seed bank formation.

In this study, we test the following hypotheses: (1) the seed bank of the rocky outcrops in the rupestrian fields in "Serra do Cipó" are both richer and denser than that formed in distinct and adjacent vegetation types and physiognomies present in the area, i.e. sandy and peaty bogs, forests, gallery forests, and "cerrados"; (2) distinct rupestrian field vegetation types (i.e., rocky outcrop, sandy and peaty bogs) are more similar to each other floristically and more distinct from the other physiognomies, because at a regional scale the general differences in seed bank size and composition inside a physiognomy or vegetation type tends to be lower than between them (e.g., WilliamsLinera 1993, Butler \& Chazdon 1998); and (3) more superficial layers would have greater seedling emergence because of the higher probability of accumulation of high seed density near the surface (e.g., Veenendaal et al. 1996).

\section{Material and methods}

Study site - The "Serra do Cipó" is located in the southern portion of the Espinhaço mountain chain in southeastern Brazil. The weather is altitudinal tropical (Cwb of Köppen; Galvão \& Nimer 1965) with warm summers and well defined dry seasons. The average annual temperature is $21.2^{\circ} \mathrm{C}$ and precipitation is $1,622 \mathrm{~mm}$ (see Madeira \& Fernandes 1999, for a review). The altitudes of the study sites varied from 800 ("cerrado") to $1,340 \mathrm{~m}$ (forest). The vegetation cover is quite diversified, varying according to the physiographic conditions (see Giulietti et al. 1987, Meguro et al. 1996). Four main physiognomies were studied (see Giulietti et al. 1987 for further details and figures 1 to 6 for illustration. Coordinates of each locality are given in table 1): (1) Rupestrian Fields - it has the greatest extension and complexity in the Espinhaço mountains; it is dominant above 1,000 m a.s.l. The rupestrian fields are the result of the quartzite erosion, forming plain areas with shallow, nutrient poor soils, with poor organic matter content. There are some vegetation types, as rocky outcrops $(\mathrm{R})$, where a typical rocky flora is found. There are also moister parts, which form permanent peaty bogs (PB), and temporary sandy bogs (SB) (Ribeiro \& Fernandes 2000). (2) "Cerrado" (C) - occurs predominately between 800 and $1,000 \mathrm{~m}$. There is a dominance of shrubbytree life forms, which declines with the increase in altitude. (3) Gallery Forests $(G)$ - found along the drainage lines,

Table 1. Location of each sample point in "Serra do Cipó", MG, Brazil. $(\mathrm{R}=$ rocky outcrop; $\mathrm{SB}=$ sandy bog; $\mathrm{PB}=$ peaty bog; $\mathrm{C}=$ "cerrado"; $\mathrm{G}=$ gallery forest; $\mathrm{F}=$ forest).

\begin{tabular}{|c|c|c|c|}
\hline Location & Altitude (m) & $\mathrm{S}$ & W \\
\hline $\mathrm{R} 1$ & 1,200 & $19^{\circ} 16^{\prime} 10^{\prime \prime}$ & $43^{\circ} 35^{\prime} 51^{\prime \prime}$ \\
\hline $\mathrm{R} 2$ & 1,180 & $19^{\circ} 16^{\prime} 21^{\prime \prime}$ & $43^{\circ} 35^{\prime} 33^{\prime \prime}$ \\
\hline R3 & 1,180 & $19^{\circ} 16^{\prime} 23^{\prime \prime}$ & $43^{\circ} 35^{\prime} 43^{\prime \prime}$ \\
\hline $\mathrm{R} 4$ & 1,220 & $19^{\circ} 16^{\prime} 47^{\prime \prime}$ & $43^{\circ} 35^{\prime} 08^{\prime \prime}$ \\
\hline SB1 & 1,170 & $19^{\circ} 16^{\prime} 15^{\prime \prime}$ & $43^{\circ} 36^{\prime} 01^{\prime \prime}$ \\
\hline SB2 & 1,200 & $19^{\circ} 17^{\prime} 29^{\prime \prime}$ & $43^{\circ} 35^{\prime} 58^{\prime \prime}$ \\
\hline SB3 & 1,200 & $19^{\circ} 17^{\prime} 21^{\prime \prime}$ & $43^{\circ} 34^{\prime} 03^{\prime \prime}$ \\
\hline PB1 & 1,190 & $19^{\circ} 16^{\prime} 12^{\prime \prime}$ & $43^{\circ} 35^{\prime} 36^{\prime \prime}$ \\
\hline PB2 & 1,160 & $19^{\circ} 16^{\prime} 06^{\prime \prime}$ & $43^{\circ} 35^{\prime} 22^{\prime \prime}$ \\
\hline $\mathrm{C} 1$ & 1,200 & $19^{\circ} 16^{\prime} 49^{\prime \prime}$ & $43^{\circ} 35^{\prime} 16^{\prime \prime}$ \\
\hline $\mathrm{C} 2$ & 1,080 & $19^{\circ} 21^{\prime} 31^{\prime \prime}$ & $43^{\circ} 37^{\prime} 50^{\prime \prime}$ \\
\hline $\mathrm{C} 3$ & 800 & $19^{\circ} 16^{\prime} 43^{\prime \prime}$ & $43^{\circ} 35^{\prime} 28^{\prime \prime}$ \\
\hline $\mathrm{C} 4$ & 800 & $19^{\circ} 21^{\prime} 12^{\prime \prime}$ & $43^{\circ} 36^{\prime} 43^{\prime \prime}$ \\
\hline G1 & 1,280 & $19^{\circ} 14^{\prime} 26^{\prime \prime}$ & $43^{\circ} 30^{\prime} 18^{\prime \prime}$ \\
\hline G2 & 1,120 & $19^{\circ} 14^{\prime} 19^{\prime \prime}$ & $43^{\circ} 30^{\prime} 41^{\prime \prime}$ \\
\hline G3 & 1,200 & $19^{\circ} 15^{\prime} 33^{\prime \prime}$ & $43^{\circ} 33^{\prime} 02^{\prime \prime}$ \\
\hline G4 & 1,170 & $19^{\circ} 16^{\prime} 24^{\prime \prime}$ & $43^{\circ} 35^{\prime} 35^{\prime \prime}$ \\
\hline F1 & 1,340 & $19^{\circ} 13^{\prime} 08^{\prime \prime}$ & $43^{\circ} 30^{\prime} 20^{\prime \prime}$ \\
\hline F2 & 1,340 & $19^{\circ} 14^{\prime} 27^{\prime \prime}$ & $43^{\circ} 30^{\prime} 43^{\prime \prime}$ \\
\hline F3 & 1,210 & $19^{\circ} 15^{\prime} 42^{\prime \prime}$ & $43^{\circ} 33^{\prime} 22^{\prime \prime}$ \\
\hline $\mathrm{F} 4$ & 1,270 & $19^{\circ} 16^{\prime} 25^{\prime \prime}$ & $43^{\circ} 32^{\prime} 41^{\prime \prime}$ \\
\hline
\end{tabular}



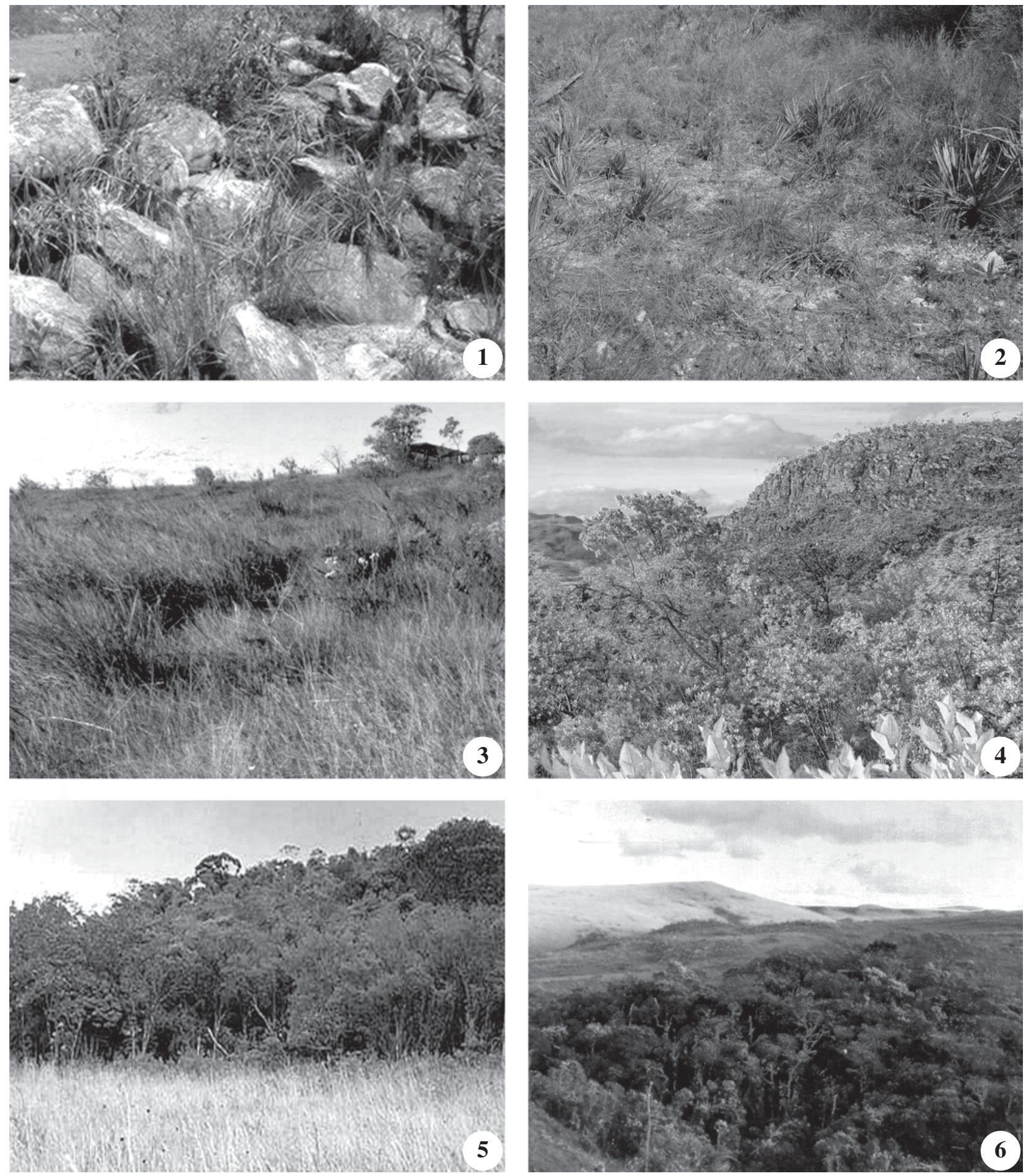

Figures 1-6. General aspect of the different vegetation types at "Serra do Cipó". 1. Rocky outcrops (R). 2. Sandy bogs (SB). 3. Peaty bogs (PB). 4. "Cerrados" (C). 5. Forests (F). 6. Gallery forests (G).

they are 5 to $10 \mathrm{~m}$ wide and reach 10 to $15 \mathrm{~m}$ of height. The gallery forests are species rich and varied. (4) Forests (F) occur as islands in the top hills without rocky boulders or outcrops and in the slopes. The soils are derived from quartzite, granite and gneiss.

Sampling - The physiognomies and vegetation types studied were located inside the conservation units of "Parque Nacional da Serra do Cipó" and the "Área de Proteção Ambiental Morro da Pedreira") and in a private protected area ("Reserva Particular Vellozia" $-19^{\circ} 17^{\prime} \mathrm{S}$ and $43^{\circ} 33^{\prime} \mathrm{W}$ ). Each physiognomy (4 types) was sampled in four different localities. We collected five soil samples with $20 \mathrm{~cm}$ diameter and $5 \mathrm{~cm}$ depth $(314$ $\mathrm{cm}^{3}$ ) from each locality of each vegetation type, to follow seedling emergence from the seed bank. Samples were 
stratified in 0 to 5 and 5 to $10 \mathrm{~cm}$, in an attempt to assess the influence of depth in the readily germinable seed bank. Sandy bogs were sampled in three localities and peaty bogs in two (table 1). In the rocky outcrops, we sampled the soil between rocks (in clay pockets). Thus, the total sample size was $n=210$ ( 21 localities $\times 5$ sample points $\times 2$ depths).

Samples were taken at the end of the dry season, in August 2002, to obtain the greatest possible number of species ready to germinate (Madeira \& Fernandes 1999, Sassaki et al. 1999, Gomes et al. 2001). In environments with well-defined seasons, as the "Serra do Cipó", many seeds have their germination stimulated by the increase in moisture (Andrade 2002). Samples were homogenized and left to germinate in punched polystyrene trays with $450 \mathrm{~cm}^{2}$ with $2 \mathrm{~cm}$ depth in a $30 \%$ shadowed greenhouse in the field. We watered the trays for 5 to 10 minutes (depending on the weather conditions) three times a day during all the study period. To avoid possible contamination with the local seed rain, we distributed trays with sand and inert substratum as control (e.g., Baider et al. 1999, 2001). The soil from trays without seedling emergence was revolved monthly to allow germination of seeds that could occur in deeper layers (Baider et al. 1999, 2001).

Seedling emergence was monitored weekly from September 2002 to August 2003. Each individual that emerged from the soil was identified at the lower possible taxonomic level. Many of them were transplanted to seedling bags with standard soils to reach a size where the identification could be possible.

Although the used method of seedling emergence monitoring underestimates the composition and density of the seed bank, it provides the readily germinable fraction and the first one to establish after a disturbance (Kirkman \& Sharitz 1994). In other words, it shows the initial natural regenerative potential of the soil. This method has been widely used because it is easier to identify seedlings than seeds and because a greater soil volume can be analyzed (Brown 1992).
To investigate the origin and geographic distribution of the emergent species, and unveil the relative importance of the species ready to germinate from the seed bank in rock outcrops, species were classified into four groups: 1 . native but restrict to "Serra do Cipó" (extinction threatened); 2. native common, wide distribution across the Espinhaço mountain chain; 3 . pantropical; and 4. cosmopolitan, invasive in "Serra do Cipó".

Statistical analysis - We compared seedling abundance, species richness, diversity (Shannon-Wiener index: $H^{\prime}=-\Sigma p i * l n_{p i}$ ), and evenness $\left(H^{\prime} \ln _{S}^{-1}\right)$ within and between physiognomies/ vegetation types by analysis of variance (ANOVAs) with $a$ posteriori Tukey test (Magurran 1988, Sokal \& Rohlf 1995). The differences in these parameters between depths were tested by paired t-tests (Sokal \& Rohlf 1995). All data were log transformed $(\log x+1)$ to reach the normality and variance homogeneity.

The similarity of species composition between the different vegetation types and localities was verified by a cluster grouping analysis with Sorensen index and centroid method to presence/absence data (Magurran 1988) and the Ward method with Euclidian distance to species abundance data (Shepherd 1994). The Ward method produces more compact and distinct groups, which facilitates the interpretation of the results (Shepherd 1994). We only used the species with doubtless identification, or those one certainly separated into specific morphospecies, with at least two individuals.

\section{Results}

Contrary to our initial expectation, rocky outcrops presented the lower abundance and richness of seeds in the soil (table 2): "cerrados", forests and gallery forests all had more seedling abundance and more species (12, 6 , and 2 times more, respectively) than the rock outcrops. Thus, the seed banks of the "cerrados" of "Serra do Cipó" ranked among those studied with highest seed

Table 2. Differential seedling abundance, species richness, diversity and evenness of the vegetation types and physiognomies in "Serra do Cipó". Values shown are not log transformed. Different letters indicate statistically significant differences at the $P$-level of 0.05 (a posteriori Tukey test). $H^{\prime}=$ Shannon-Wiener diversity indices $=-\Sigma p i^{*} / n_{p i}$ Evenness $=H^{\prime} / l n_{s}$. Data shown are not log transformed.

\begin{tabular}{|c|c|c|c|c|}
\hline \multirow{2}{*}{ Vegetation type } & Seedling abundance & Species richness & \multirow{2}{*}{$H^{\prime}$} & \multirow{2}{*}{ Evenness } \\
\hline & \multicolumn{2}{|c|}{ (average $\pm \mathrm{s} /$ range: $\min -\max$ ) } & & \\
\hline Rocky outcrops & $19.25^{\mathrm{a}} \pm 20.77 / 1-26$ & $8.25^{\mathrm{a}} \pm 6.55 / 1-18$ & $1.60^{\mathrm{a}} \pm 1.10$ & $0.69^{\mathrm{a}} \pm 0.40$ \\
\hline Sandy bogs & $47.67^{a} \pm 63.36 / 24-1119$ & $6.33^{a} \pm 7.51 / 4-22$ & $0.87^{\mathrm{a}} \pm 0.79$ & $0.64^{\mathrm{a}} \pm 0.30$ \\
\hline Peaty bogs & $93 \pm 125.86 / 23-563$ & $21 \pm 26.87 / 4-53$ & $2.15 \pm 2.06$ & $0.99^{\mathrm{b}} \pm 0.01$ \\
\hline "cerrados" & $\pm 207 \quad / 65-752$ & $25.25^{b} \pm 10.34 / 18-36$ & \pm 0.32 & $0.72 \pm 0.17$ \\
\hline Gallery forests & $\pm 112.09 / 57-346$ & $17.75^{b} \pm 6.24 / 18-39$ & $2.30 \pm 0.39$ & $0.82 \pm 0.17$ \\
\hline Forests & $126.75^{b} \pm 100.26 / 40-293$ & $20.25^{b} \pm 6.85 / 20-39$ & $2.44^{b} \pm 0.30$ & $0.83^{\mathrm{b}} \pm 0.13$ \\
\hline$F_{(5,57)}$ & 7.602 & 7.309 & 3.316 & 2.969 \\
\hline$P$ & 0.0001 & 0.0001 & 0.0145 & 0.0241 \\
\hline
\end{tabular}


abundance and species richness. Sandy bogs followed a pattern similar to the rock outcrops. The gallery forests showed the greatest variation in seedling abundance and species richness (table 2).

Species similarity between vegetation types and physiognomies was low (17\% for abundance and $44 \%$ for presence/absence data; figure 7). Rocky outcrop sites were not grouped, as happened for the most part of vegetation types or physiognomies. Exceptions are the high similarity between the "cerrados" 2 and 4 and between the sandy bogs 1 and 2 . The rocky outcrops tended to be isolated from the other physiognomies and vegetation types, indicating the peculiarity of its flora and its great variability in seed bank species composition.
Six Melastomataceae species emerged from the rocky outcrop soils (Marcetia taxifolia, two Miconia, two Tibouchina species, and one undetermined species; table 3). Considering all physiognomies, Melastomataceae was the second most species rich family, with 22 morphospecies (table 3); Asteraceae ranked as the family richest in species, with 23 morphospecies identified. It was more representative in "cerrado" $(22.2 \%$ of all emergent species) and gallery forests (25.6\%), but also occurred in all other physiognomies. Only two Leguminosae species emerged, but not from rupestrian fields soils (one in "cerrado" and one in forest). Melastomataceae was the richest family in rocky outcrops, representing $38.9 \%$ of all species that emerged,
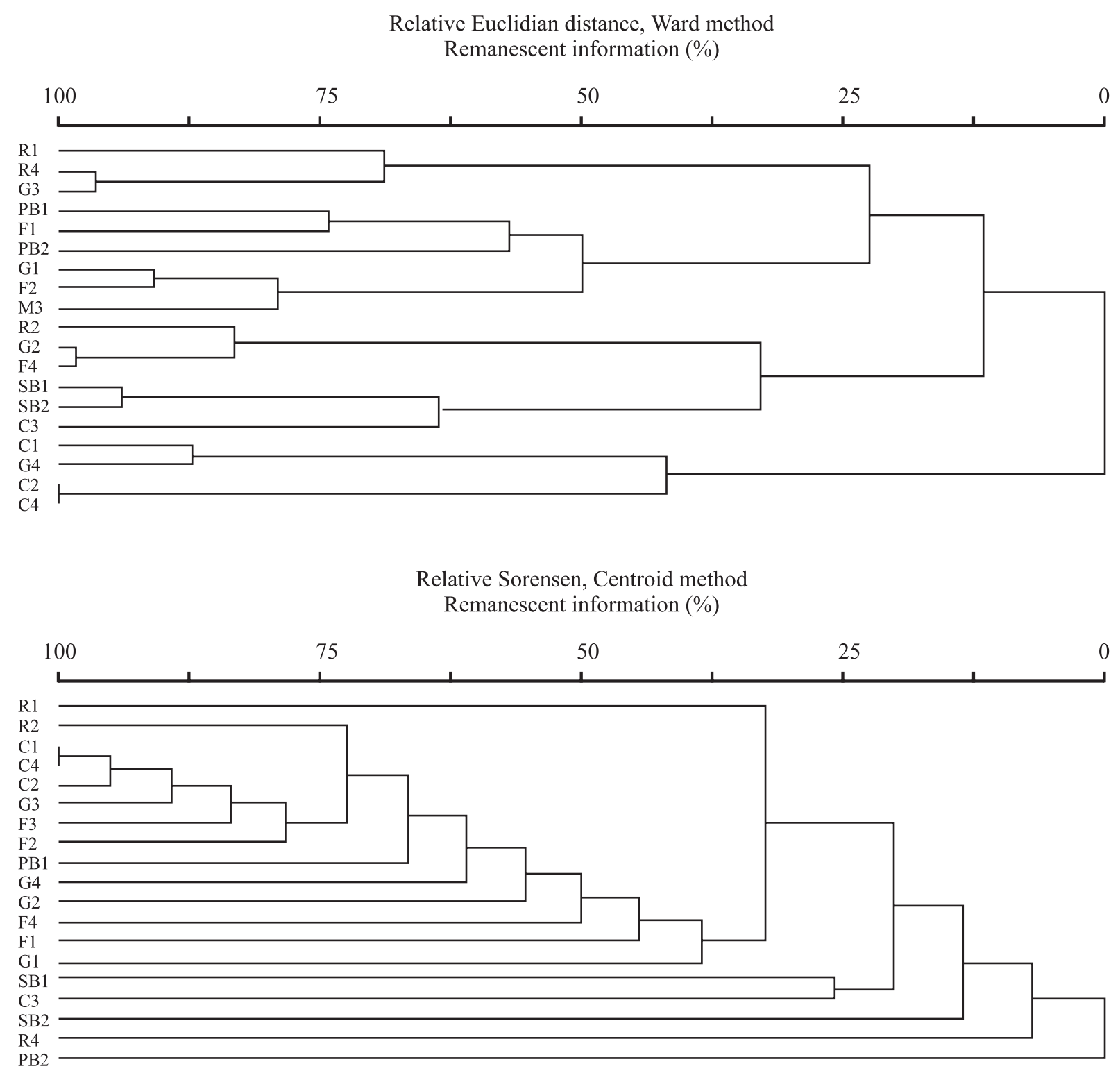

Figure 7. Cluster analyses with a) abundance and $\mathrm{b}$ ) presence / absence data. ( $\mathrm{R}=$ rocky outcrops, $\mathrm{SB}=$ sandy bogs, $\mathrm{PB}=$ peaty bogs, $\mathrm{C}=$ "cerrados", $\mathrm{G}=$ gallery forests, $\mathrm{F}=$ forests). 
Table 3. Plant species and families that germinated from the soil seed banks in "Serra do Cipó", MG, Brazil. Geographic distribution patterns (GD): 1. restricted native (extinction threatened), 2. common native, distributed across Espinhaço mountain chain, 3. pantropical, 4. cosmopolitan invasive. The values in each vegetation type or physiognomy, refers to the abundances in each vegetation type $(\mathrm{R}=$ rocky outcrops, $\mathrm{SB}=$ sandy bogs, $\mathrm{PB}=$ peaty bogs, $\mathrm{C}=$ "cerrados", $\mathrm{G}=$ gallery forests, $\mathrm{F}=$ forests).

\begin{tabular}{|c|c|c|c|c|c|c|c|c|c|}
\hline Family & Species & $\mathrm{R}$ & SB & PB & $\mathrm{C}$ & G & $\mathrm{F}$ & GD & References \\
\hline AQUIFOLIACEAE & Ilex sp. & & & & & 1 & & & \\
\hline ASTERACEAE & $\begin{array}{l}\text { Acanthospermum australe } \\
\text { (Loefl.) Kuntze }\end{array}$ & & & & 1 & & & 3 & Lorenzi (2000) \\
\hline \multirow[t]{23}{*}{ (23 morfospecies) } & $\begin{array}{l}\text { Ageratum myriadenium } \\
\text { (Sch. Bip. ex Baker) }\end{array}$ & 1 & & 2 & 198 & 5 & 1 & 3 & \\
\hline & R.M. King \& H. Rob. & & & & & & & & M. Sobral (unpublished data) \\
\hline & Ageratum sp.1 & & & & 3 & & & & \\
\hline & Ageratum sp.2 & & & 6 & & & & & \\
\hline & Ageratum sp. 3 & 2 & & & & & & & \\
\hline & Aspilia sp. & & & & 2 & & & & \\
\hline & Baccharis sp. & & & & & 2 & & & \\
\hline & Crysolaena sp. & & & 1 & & 18 & 10 & & \\
\hline & $\begin{array}{l}\text { Cyrtocymura scorpioides } \\
\text { (Lam.) H. Rob. }\end{array}$ & & & & & & 1 & 3 & Robinson (1999) \\
\hline & $\begin{array}{l}\text { Eremanthus cf. incanus } \\
\text { (Less.). Less. }\end{array}$ & & & & & & 97 & 2 & Rodrigues et al. (2002) \\
\hline & Lepidaploa $\mathrm{sp} .1$ & & & & 11 & 1 & & & \\
\hline & Lepidaploa sp.2 & & & & & 1 & & & \\
\hline & Lessingianthus sp. & & & & 1 & & & & \\
\hline & Lychnophora sp. & & 2 & & & & & & \\
\hline & $\begin{array}{l}\text { Mikania micrantha }(\text { L.) } \\
\text { Kunth }\end{array}$ & & & & & 4 & & 3 & $\begin{array}{l}\text { http://www.hear.org/pier/ } \\
\text { species/mikania_micrantha. } \\
\text { htm }\end{array}$ \\
\hline & $\begin{array}{l}\text { Praxelis capillaris (DC.) } \\
\text { Sch. Bip. }\end{array}$ & 3 & & & & 1 & & 3 & M. Sobral (unpublished data) \\
\hline & Youngia japonica (L.) DC. & & & & 3 & 1 & 4 & 4 & Lorenzi (2000) \\
\hline & sp.1 & & & & 2 & 9 & 1 & & \\
\hline & sp. 2 & & & 2 & & & & & \\
\hline & sp.3 & & & 1 & & & & 1 & \\
\hline & sp.4 & & & & & & 1 & & \\
\hline & sp. 5 & & & & & & 2 & & \\
\hline & sp.6 & & & & & 6 & 1 & & \\
\hline CACTACEAE & $\begin{array}{l}\text { Pilosocereus aurisetus } \\
\text { (Werderm.) F. Buxbaum }\end{array}$ & & & & & 1 & & 1 & Zappi (1994) \\
\hline CYPERACEAE & Bulbostilis sp. & 4 & & & & & 6 & & \\
\hline \multirow[t]{8}{*}{ (11 morphospecies) } & $\begin{array}{l}\text { Cyperus hermaphroditus } \\
\text { Standl. }\end{array}$ & & & & & 1 & 3 & 3 & $\begin{array}{l}\text { http://biblio68.ibiologia. } \\
\text { unam.mx/FullText/lfl6.html }\end{array}$ \\
\hline & Cyperus niger R. \& P. & 2 & 12 & 27 & 20 & 4 & 1 & 3 & $\begin{array}{l}\text { http://www.puc.edu/Faculty/ } \\
\text { Gilbert_Muth/Cyperus.htm }\end{array}$ \\
\hline & $\begin{array}{l}\text { Fimbristylis cf. complanata } \\
\text { (Retz.) Link }\end{array}$ & & & & & 6 & & 3 & M. Sobral (unpublished data) \\
\hline & Fymbristilis sp.1 & 2 & 492 & 129 & 48 & 8 & 21 & & \\
\hline & Fymbristilis sp.2 & & & & 7 & 15 & & & \\
\hline & Fymbristilis sp.3 & & & & & 4 & & & \\
\hline & Killinga sp. & 1 & & 20 & 145 & & & & \\
\hline & $\begin{array}{l}\text { Lagenocarpus sp. } \\
\text { sp.1 }\end{array}$ & 12 & $\begin{array}{c}193 \\
67\end{array}$ & 7 & 29 & & 8 & & \\
\hline
\end{tabular}


continuation

\begin{tabular}{|c|c|c|c|c|c|c|c|c|c|}
\hline Family & Species & $\mathrm{R}$ & SB & PB & $\mathrm{C}$ & G & $\mathrm{F}$ & GD & References \\
\hline \multirow[t]{3}{*}{ ERIOCAULACEAE } & Leyothrix sp. & & 1 & & & & & & \\
\hline & $\begin{array}{l}\text { Leyothrix or Paepalanthus } \\
\text { sp.1 }\end{array}$ & & & 2 & & & & & \\
\hline & $\begin{array}{l}\text { Leyothrix or Paepalanthus } \\
\text { sp. } 2\end{array}$ & & 4 & & & & & & \\
\hline \multirow[t]{3}{*}{ EUPHORBIACEAE } & $\begin{array}{l}\text { Croton urucurana or } \\
\text { floribundus }\end{array}$ & & & & & 1 & & 2 & $\begin{array}{l}\text { server.propp.ufms.br/ } \\
\text { producao/ceuldin.htm }\end{array}$ \\
\hline & $\begin{array}{l}\text { Phyllanthus minutulus } \\
\text { Muell. Arg. }\end{array}$ & & & & 8 & & & 1 & Cordeiro (1992) \\
\hline & $\begin{array}{l}\text { Sebastiania hispida } \\
\text { (Mart.) Pax }\end{array}$ & & & & 1 & & & 3 & $\begin{array}{l}\text { www.goacom.com/ } \\
\text { goafoundation/biodiversity/ } \\
\text { annexure6c.htm }\end{array}$ \\
\hline GENTIANACEAE & Schultesia gracilis Cham. & & & 7 & & & & 2 & $\begin{array}{l}\text { E. Guimarães (unpublished } \\
\text { data); www.semarth.df.gov. } \\
\text { br/site.cap15/01.htm }\end{array}$ \\
\hline IRIDACEAE & sp.1 & & 17 & 1 & & 1 & & & \\
\hline LAMIACEAE & Hyptis sp. & 5 & & & 1 & & 2 & & \\
\hline \multirow[t]{2}{*}{ LEGUMINOSAE CAES. } & $\begin{array}{l}\text { Chamaecrista dentata } \\
\text { (Vogel) H.S.Irwin \& Barneby }\end{array}$ & & & & & & 2 & 1 & $\begin{array}{l}\text { http://www.biodiversitas.org. } \\
\text { brlfloramglframe_flora.htm }\end{array}$ \\
\hline & $\begin{array}{l}\text { Chamaecrista desvauxii } \\
\text { (Colladon) Killip }\end{array}$ & & & & 7 & & & 3 & $\begin{array}{l}\text { www.ildis.org/Legume Web/ } \\
6.00 / \text { taxa/113308.shtml }\end{array}$ \\
\hline LYTHRACEAE & $\begin{array}{l}\text { Diplusodon orbicularis } \\
\text { Koehne }\end{array}$ & 11 & & 3 & & 1 & & 1 & Cavalcanti (1990) \\
\hline MALVACEAE & Sida glaziovii K. S. Schum. & & & & 286 & & & 3 & Lorenzi (2000) \\
\hline \multirow{4}{*}{$\begin{array}{l}\text { MELASTOMATACEAE } \\
\text { ( } 22 \text { morfospecies) }\end{array}$} & $\begin{array}{l}\text { Cambessedesia hilariana } \\
\text { (A. St.-Hil. ex Bonpl.) DC. }\end{array}$ & & 4 & 7 & & & & 2 & $\begin{array}{l}\text { D. Negreiros } \\
\text { (unpublished data) }\end{array}$ \\
\hline & Lavoisiera confertifolia Cogn. & & 10 & & & & & 2 & $\begin{array}{l}\text { D. Negreiros } \\
\text { (unpublished data) }\end{array}$ \\
\hline & $\begin{array}{l}\text { Lavoisiera cordata or } \\
\text { campos-portoana Cogn. }\end{array}$ & 2 & & & 3 & & & 1 & $\begin{array}{l}\text { http://www.biodiversitas.org. } \\
\text { brlfloramglframe_flora.htm }\end{array}$ \\
\hline & Marcetia taxifolia Cogn. & 3 & 57 & & 1 & 6 & & 2 & $\begin{array}{l}\text { Romero \& Martins (2002) } \\
\text { www.nybg.org/bsci/hcol/ } \\
\text { vasc/melastomataceae.html }\end{array}$ \\
\hline
\end{tabular}

Miconia sp.1

Miconia sp.2

Miconia sp.3

Miconia sp.4

Microlicia sp.1

Microlicia sp.2

Microlicia sp.3

Microlicia sp.4

Microlicia sp.5

Microlicia sp.6

Tibouchina sp.1

Tibouchina sp.2

Tibouchina sp.3

Tibouchina sp.4

Tibouchina sp.5

sp. 1

sp. 2

sp. 3

4

4
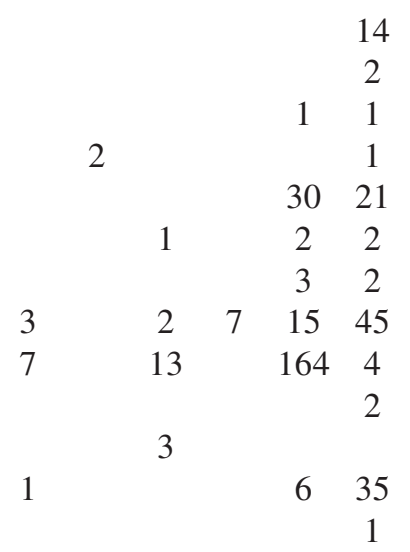
continuation

\begin{tabular}{|c|c|c|c|c|c|c|c|c|c|}
\hline Family & Species & $\mathrm{R}$ & SB & $\mathrm{PB}$ & $\mathrm{C}$ & G & $\mathrm{F}$ & GD & References \\
\hline MYRTACEAE & sp1 & & & & 23 & & & & \\
\hline \multirow[t]{2}{*}{ OCHNACEAE } & Sauvagesia erecta $\mathrm{L}$. & & & 54 & & 2 & 6 & 3 & $\begin{array}{l}\text { E. Guimarães } \\
\text { (unpublished data) }\end{array}$ \\
\hline & Sauvagesia sp. & 1 & & 1 & & 6 & & & \\
\hline POACEAE & $\begin{array}{l}\text { Axonopus capillaris (Lam.) } \\
\text { Chase }\end{array}$ & & & & 11 & & & 3 & $\begin{array}{l}\text { http://www.inbio.ac.cr/bims/ } \\
\text { k03/p13/c046/o0159/f01382/ } \\
\text { g008604/s027163.htm }\end{array}$ \\
\hline \multirow[t]{10}{*}{ (10 morfospecies) } & & & & & 19 & & & 3 & $\begin{array}{l}\text { http://plants.usda.gov/cgi_ } \\
\text { bin/plant_profile.cgi? } \\
\text { symbol=DIFI; }\end{array}$ \\
\hline & $\begin{array}{l}\text { Digitaria filiformis (L.) } \\
\text { Koel. }\end{array}$ & & & & & & & & $\begin{array}{l}\text { http://148.233.168.204/pfnm/ } \\
\text { DigitariaFiliformis.html }\end{array}$ \\
\hline & $\begin{array}{l}\text { Eragrostis articulata } \\
\text { (Schrank) Nees }\end{array}$ & 231 & & 11 & & & & 3 & $\begin{array}{l}\text { Boechat \& Longhi-Wagner } \\
\text { (2000) }\end{array}$ \\
\hline & $\begin{array}{l}\text { Eragrostis maypurensis } \\
\text { (HBK) Steud. }\end{array}$ & & & 231 & & 11 & & 3 & $\begin{array}{l}\text { Boechat \& Longhi-Wagner } \\
(2000)\end{array}$ \\
\hline & Ichnanthus sp. & & & 18 & 1 & 21 & 32 & & \\
\hline & Panicum parviflorum $\mathrm{R} . \mathrm{Br}$. & & & 6 & & & & 3 & $\begin{array}{l}\text { http://www.inhs.uiuc.edu/ } \\
\text { cbd/collections/botany/ } \\
\text { endanger.html }\end{array}$ \\
\hline & Panicum pilosum $\mathrm{Sw}$. & & & & & 5 & 1 & 3 & $\begin{array}{l}\text { http://scisun.nybg.org:8890/ } \\
\text { searchdb/owa/www. } \\
\text { specimen.search_list?taxon= } \\
\text { Panicum\&projcode=GUIA }\end{array}$ \\
\hline & Paspalum sp. & & & & 52 & & & & \\
\hline & sp.1 & 54 & 130 & 142 & 194 & 98 & 19 & & \\
\hline & sp.2 & 4 & 7 & 65 & 17 & 7 & 10 & & \\
\hline POLYGALACEAE & $\begin{array}{l}\text { Polygala herbicola A. } \\
\text { St.-Hil. }\end{array}$ & & & 1 & & & & 3 & Marques (1998) \\
\hline \multirow[t]{2}{*}{ PORTULACACEAE } & Portulaca oleracea & & & & 20 & & 4 & 4 & $\begin{array}{l}\text { Jorgensen \& Ulloa (1994), } \\
\text { Lorenzi (2000) }\end{array}$ \\
\hline & Portulaca sp. & & & & & 4 & & & \\
\hline RUBIACEAE & $\begin{array}{l}\text { Borreria capitata (Ruiz \& } \\
\text { Pav.) DC. }\end{array}$ & & & & 50 & 2 & 16 & 3 & $\begin{array}{l}\text { www.cac.br/relatorios/ } \\
\text { herbario/Igenero.asp?tarefa= } \\
\text { borreria }\end{array}$ \\
\hline \multirow[t]{5}{*}{ (6 morfospecies) } & Borreria verticillata Mayer & & & 91 & & & & 3 & $\begin{array}{l}\text { www.ib.usp.br/ } \\
\text { beeplant.bove.htm }\end{array}$ \\
\hline & Borreria sp. & & & & & & 1 & & \\
\hline & Coccocypselum sp. & & & 5 & & & & & \\
\hline & Psyllocarpus sp. & & 1 & & & & & & \\
\hline & $\begin{array}{l}\text { Richardia brasiliensis } \\
\text { Gomes }\end{array}$ & & & & 19 & & & 3 & Lorenzi (2000) \\
\hline SOLANACEAE & $\begin{array}{l}\text { Schwenkia americana L. } \\
\text { Solanum sp. }\end{array}$ & & & 1 & & & 1 & 3 & L. Freire (unpublished data) \\
\hline
\end{tabular}

and also in sandy bogs (18.2\%) and in forests (33.3\%) Melastomataceae represented $26 \%$ of the species that emerged from rupestrian field soils (i.e., rocky outcrops + bogs).

Seedling emergence was significantly higher in the superficial layers $(0-5 \mathrm{~cm})$ of all studied vegetation types, except for the bogs (table 4, figure 8). The difference was more pronounced in the rocky outcrops, where the emergence in the more superficial layers was approximately 12 times greater than the deeper layers (table 4).

The rocky outcrops contained the largest proportion of restricted native threatened species $(14.3 \%$, e.g. 

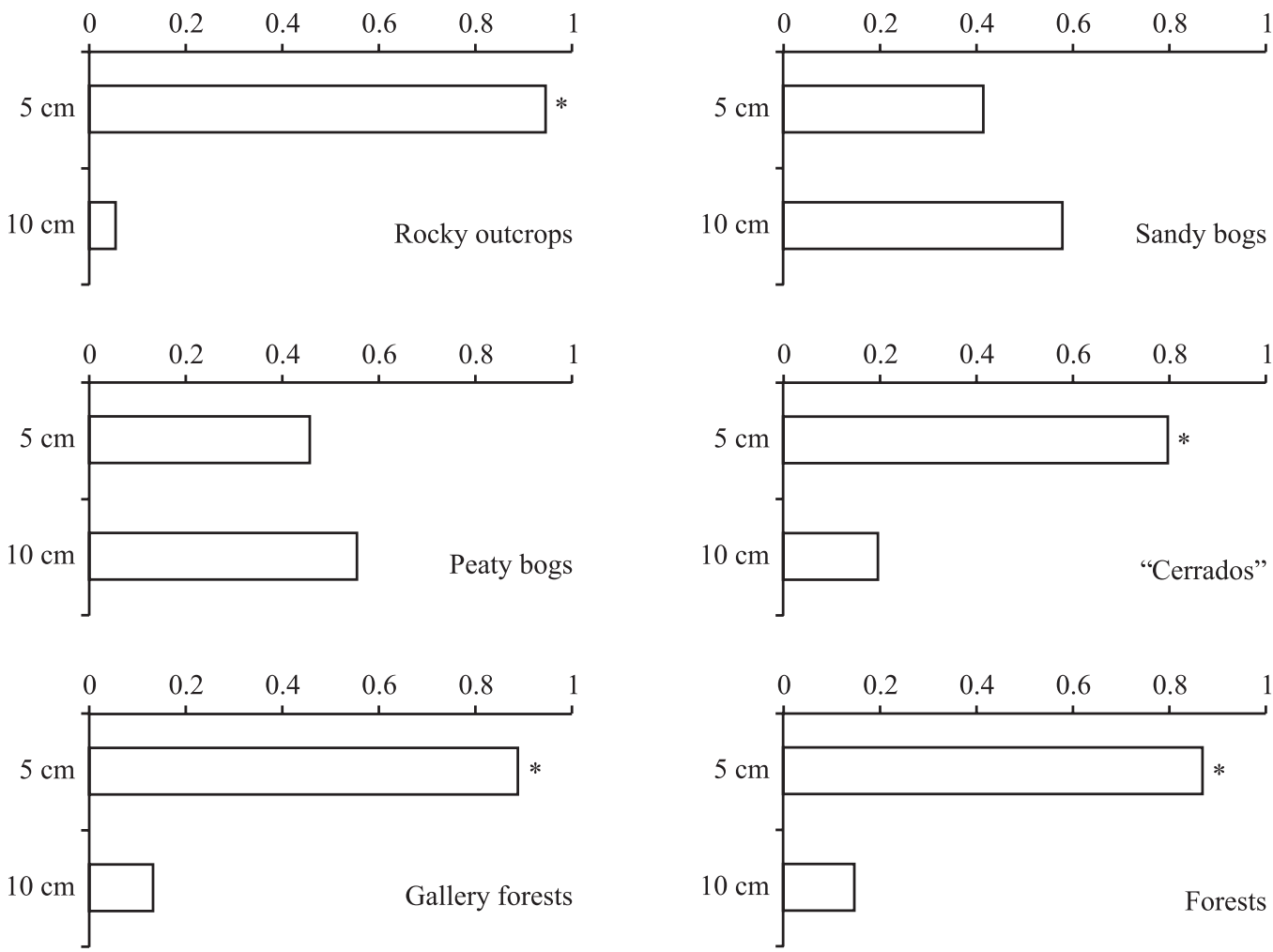

Figure 8. Emergent seedling proportion in each soil depth layer at each vegetation type. Asterisks indicate statistically significant difference at $0.05 P$ level (paired t test).

Table 4. Seedling abundance and emergent species richness between soil depths of each vegetation type compared by paired t test (Average \pm standard deviation). Data shown are not log transformed.

\begin{tabular}{|c|c|c|c|c|c|c|c|c|}
\hline \multirow{2}{*}{ Vegetation type } & \multicolumn{4}{|c|}{ Abundance } & \multicolumn{4}{|c|}{ Richness } \\
\hline & $5 \mathrm{~cm}$ & $10 \mathrm{~cm}$ & $\mathrm{t}$ & $P$ & $5 \mathrm{~cm}$ & $10 \mathrm{~cm}$ & $\mathrm{t}$ & $P$ \\
\hline Rocky outcrops & $0.38 \pm 0.47$ & $0.03 \pm 0.09$ & 10.68 & 0.0023 & $0.29 \pm 0.32$ & $0.03 \pm 0.09$ & 11.57 & 0.0016 \\
\hline Sandy bogs & $0.72 \pm 0.41$ & $0.91 \pm 0.37$ & 0.14 & 0.7068 & $0.43 \pm 0.11$ & $0.49 \pm 0.23$ & 0.00 & 0.9511 \\
\hline Peaty bogs & $1.25 \pm 0.58$ & $1.43 \pm 0.71$ & 0.32 & 0.5794 & $0.91 \pm 0.63$ & $1.01 \pm 0.59$ & 0.11 & 0.7443 \\
\hline “Cerrados" & $1.22 \pm 0.37$ & $0.47 \pm 0.29$ & 10.41 & 0.0026 & $0.67 \pm 0.30$ & $0.23 \pm 0.24$ & 26.32 & 0.0001 \\
\hline Gallery forests & $0.96 \pm 0.64$ & $0.24 \pm 0.39$ & 16.85 & 0.0002 & $0.55 \pm 0.34$ & $0.17 \pm 0.25$ & 15.25 & 0.0004 \\
\hline Forests & $1.12 \pm 0.56$ & $0.39 \pm 0.38$ & 22.52 & 0.0001 & $0.56 \pm 0.23$ & $0.30 \pm 0.28$ & 10.22 & 0.0028 \\
\hline
\end{tabular}

Diplusodon orbicularis, Marcetia taxifolia, Lavoisiera cordata or L. campos-portoana). The sandy bogs showed the greatest proportion (60\%) of native species distributed along the Espinhaço mountain chain (figure 9, table 3).

The most abundant emergent species in rocky outcrops had wide geographic distribution (71.4\%), and the same pattern occurred in all physiognomies (varying from $40 \%$ in sandy bogs to $75 \%$ in "cerrados"; figure 9, table 3). Examples of such species are Ageratum myriadenium, Mikania micrantha, Cyperus niger, Chamaecrista desvauxii, Sebastiania hispida, Sida glaziovii, Digitaria filiformis, Polygala herbicola, Borreria capitata and Schwenkia americana (table 3 ).

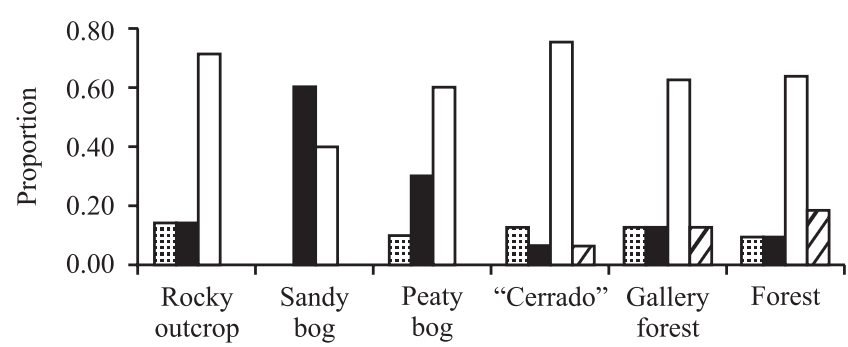

Figure 9. Porportion of species in each geographical distribution class per physiognomy and vegetation type. Restricted native and extinction threatened (田), common native, distributed across Espinhaço mountain chain (ם), Pantropical ( $\square$ ) and cosmopolitan invasive ( $\square$ ). 


\section{Discussion}

Despite the typically high species richness of the mature vegetation in the rupestrian fields (i.e., rocky outcrops and bogs) of "Serra do Cipó" (Giulietti et al. 1987), they had the seed banks which were poorest in species and less dense, when compared to neighboring "cerrado", forests, and gallery forest. The rocky outcrops, in particular, were poorer in species also when compared to similar environments ( 35 species - Houle \& Phillips 1988; 43 species - Holmes \& Cowling 1997). The prevalence of moisture extremes and the dry conditions, the predominance of species with passive dispersal syndrome, we propose, are among the causal factors behind this pattern.

The low abundance of emergent seedlings and species richness in these vegetation types can be attributed to high seed dormancy and germination inhibition, which leads to low germination rates (Houle \& Philips 1988, Leck \& Brock 2000). High dormancy is possibly due to the high moisture extremes of the rocky outcrops and sandy bogs, which can be very dry or very humid, depending on the rainfall regime. The dry conditions are fundamental to increase the ability of seeds to stay dormant (Brock et al. 2003) and the photoinduction of germination can be limited in microhabitats with extremely dry conditions (Gallagher \& Cardina 1997). Germaine \& McPherson (1999) also verified lower emergence in sandier environments, compared to forest physiognomies. Thus, although there is a high probability of elevated species number in the seed banks of rupestrian fields, both the species richness and abundance of emergent seedlings are normally low (Leck \& Brock 2000).

On the other hand, the temporary very humid conditions in rocky outcrops and sandy bogs contribute to a random distribution of groups of seed (Houle \& Philips 1988, Chambers 1993, Yu et al. 2003), because there is a great proportion of species with passive dispersal syndromes, mainly by water (Bigwood \& Inouye 1988), which normally accumulates in specific microsites. This characteristic reduces the quality of sampling. So, there is a probability of underestimating the real readily germinable seed bank, in addition to the high dormancy and tendency to germination inhibition of the majority of the species. Differences in seedling emergence between physiognomies are often attributed to differences in species persistence in the seed bank and disturbance regimes (e.g., Thompson \& Grime 1979). For instance, the greater seedling abundance and species richness in forest and "cerrado" formations, comparing to the rocky outcrop, might be related to the normally great germination of their seed bank after disturbances (Vázquez-Yanes \& Orozco-Segovia 1993, Veenendaal et al. 1996, Holl 1999, Pearson et al. 2002, Setterfield 2002). In the case of the gallery forests, they are often heterogeneous due to variations in water table depths, nutrient lixiviation, and erosion, which result in spatial differences in fertility (Fonseca \& Walter 2001). These three processes that occur at soil level were not yet addressed in "Serra do Cipó". Since they directly affect seed moisturing and gas exchange as well as the temperature and light regime of the microhabitats (Sousa-Silva et al. 2001), seed germination, density and number of seedlings tend to be variable in the landscape, as found here.

The similarity analysis showed the isolation of the rocky outcrops in relation to themselves and in relation to all physiognomies, as well as the rupestrian fields' vegetation types. This demonstrates its heterogeneity and uniqueness in species composition of its seed banks. Therefore, any serious impact in a particular rocky outcrop in "Serra do Cipó" can negatively influence the overall species diversity of these rupestrian fields, because endemic threatened species might occur only on it.

The patterns of seed bank similarity in species composition can be explained by factors such as specific requirements for germination, geographic proximity, physiognomic characteristics and relations with the mature vegetation (Garwood 1989, Williams-Linera 1993, Butler \& Chazdon 1998, Dupuy \& Chazdon 1998, Silva Júnior et al. 2001). For instance, the grouping found for some "cerrado" and bog localities can reflect specific requirements for germination. Garwood (1989) has shown that savannah annuals often explore neighbouring seasonally flooded areas to germinate and reproduce before the water drains off. Also, the greater temperature fluctuations and lower humidity levels in "cerrados" can explain its separation from forests because these factors inhibit the germination of some forest species. The geographic proximity to other communities is arguably an important factor in the determination of seed bank species composition in gallery forests (Silva Júnior et al. 2001) and tropical forests (Dupuy \& Chazdon 1998), which might explain, for example, the relatively high similarity between gallery forest 1 and forest 2 . The high similarity between sandy bogs 1 and 2 can be due to the physiognomic similarity, as well as geographic proximity.

The marked presence of Melastomataceae species in the rupestrian fields' and forests' seed banks is in accordance to the wide occurrence of this family in the tropics. Melastomataceae has many pioneer species (Ellison et al. 1993). For instance, the genera Miconia 
and Tibouchina, which emerged in all physiognomies, have small seeds and photoblastically positive species (Pearson et al. 2002) with high dormancy and fast germination after disturbance. These are common genera in the seed banks and in early regeneration in tropical forests and savannas (Sassaki et al. 1999, Dupuy \& Chazdon 1998, Baider et al. 1999, Andrade 2002). The genera of Melastomataceae that emerged from the seed banks of rupestrian fields in "Serra do Cipó" are quite common also in the corresponding mature vegetation (Souza 1997), perhaps resulting in their abundance in our study. The seed availability in the mature vegetation is an important factor in the seed bank composition (Houle \& Philips 1988, Willems 1995, Aziz \& Khan 1996, Jensen 1998, Guardia et al. 2000, Erschbamer et al. 2001) and determines the difference between physiognomies (Holl 1999).

The same pattern is seen for the Asteraceae, which is the richest plant family in the flora of "Serra do Cipó" (Giulietti et al. 1987), had the greatest number of species emerging from the "cerrado" and forests soils studied, and was present in all physiognomies. However, the low emergence of Leguminosae species, which is a very species-rich family in the rupestrian fields' mature vegetation (Giulietti et al. 1987), represents an opposite trend. Many species of the Leguminosae need specific mechanisms to stimulate seed germination (e.g., Gomes et al. 2001, Silveira et al. 2005), which could contribute to the low emergence observed.

In all physiognomies and vegetation types studied here, except the bogs, seed germination was higher in the more superficial soils. This pattern is widely reported for many tropical systems (e.g., Veenendaal et al. 1996, Sassaki et al. 1999, Andrade 2002). It might result from a negative influence of depth on the detection of germination stimuli (Pearson et al. 2002), to the greater probability of seed concentration near the surface, and/or to a decrease of nutrient concentration with depth (Sassaki et al. 1999, B. Medina unpublished data). Bogs were the exception to this trend and, as reported elsewhere for bogs and sandy environments (Leck 1989, Gunster 1994, Bowers 1996), showed no relationship between seedling emergence and seed distance from the soil surface. Indeed, Leck (1989) has argued that water mechanical action can have a fundamental influence in seed localization in the deeper layers, mainly for the smaller ones. As a result, soil profiles of flooded habitats tend to show large variation in seed density or germination with depth.

The expressive proportion of restricted native threatened species in rocky outcrops indicate the importance of its seed banks for conservation of the endemic flora of "Serra do Cipó" rupestrian fields, despite of the low emergence observed. The high proportion of restricted native threatened species can be attributed to the mechanic disturbance we caused, which is not a common disturbance in this environment. Rare and unpredictable events could increase the native species contribution in regeneration from the soil bank (Masaki et al. 1998, Moles \& Drake 1999).

The dominance of species with pantropical distribution in all physiognomies demonstrates their ability to germinate, emerge and perhaps establish after a disturbance, which is itself an explanation to its successful widespread distribution (Begon et al. 1996).

Our results indicate that although forests and "cerrado" physiognomies presented more potential for regeneration through seeds germination from seed banks, the rupestrian fields' vegetation types, specially the rocky outcrops, store very important species for its conservation, such as endemic and rare plants. More studies are needed to understand the specific mechanisms for germination stimuli of these species, for management purposes.

Acknowledgements - We thank F. Silveira, C. Louzada, D. Negreiros and Y. Rhoessler for help in the soil samples collection in the field; E. Almada, G. Maciel and F. Machado for help in the seedling emergence monitoring; K. T. Ribeiro for providing information about the "Parque Nacional da Serra do Cipó"; M. A. Fontes for statistical support and suggestions in the discussion; M. Sobral, J. Stehman, P. Viana, J. Ordones, E. Guimarães, M. C. Marques, L. Freire for plant identifications; F.R. Scarano and two anonymous reviewers for important suggestions and criticism; Fapemig (CRA 388/01, 583/03, 697/06), CNPq (47.9684/2001-4, 47.2491/2003-2, 304851/ 2004-3), Planta Tecnologia Ambiental and US Fish and Wildlife Service for funding. This study was part of a Master thesis of the Ecology, Conservation and Wild Life Management at the "Universidade Federal de Minas Gerais".

\section{References}

ABREU, M.E.P. \& GARCIA, Q.S. 2005. Efeito da luz e da temperatura na germinação de sementes de quatro espécies de Xyris ocorrentes na Serra do Cipó (MG). Acta Botanica Brasilica 19:149-154.

ANDRADE, L.A.Z. 2002. Impacto do fogo no banco de sementes de cerrado sensu stricto. Tese de doutorado, Universidade de Brasília.

AZIZ, S. \& KHAN, M. A. 1996. Seed bank dynamics of a semi-arid coastal shrub community in Pakistan. Journal of Arid Environments 34:81-87.

BAIDER, C., TABARELLI, M. \& MANTOVANI, W. 1999. O banco de sementes de um trecho de floresta atlântica montana (São Paulo, Brasil). Revista Brasileira de Biologia 59:319-328. 
BAIDER, C., TABARELLI, M. \& MANTOVANI, W. 2001. The soil seed bank during Atlantic forest regeneration in southeast Brazil. Revista Brasileira de Biologia 61:35-44.

BAKER, H.G. 1989. Some aspects of the natural history of seed banks. In Ecology of soil seed banks (M.A. Leck, V.T. Parker \& R.L Simpson, eds.). Academic Press, San Diego, p.9-21.

BEGON, M., HARPER, J.L. \& TOWNSEND, C.R. 1996. Ecology: individuals, populations and communities. Blackwell, London.

BIGWOOD, D.W. \& INOUYE, D. 1988. Spatial patterns analysis of seed banks: an improved method and optimized sampling. Ecology 69:497-507.

BOECHAT, S.C. \& LONGHI-WAGNER, H.M. 2000. Padrões de distribuição geográfica dos táxons brasileiros de Eragrostis (Poaceae, Chloridoideae). Revista Brasileira de Botânica 23:177-194.

BOWERS, J.E. 1996. Seedling emergence on Sonoran Desert dunes. Journal of Arid Environments 33:63-72.

BROCK, M.A., NIELSEN, D.L., SHIEL, R.J., GREEN, J.D. \& LANGLEY, J.D. 2003. Drought and aquatic community resilience: the role of eggs and seeds in sediments of temporary wetlands. Freshwater Biology 48:1207-1218.

BROWN, D. 1992. Estimating the composition of a forest seed bank: a comparison of the seed extraction and seedling emergence methods. Canadian Journal of Botany 70:1603-1612.

BUTLER, B.J. \& CHAZDON, R.L. 1998. Species richness, spatial variation, and abundance of the soil seed bank of secondary tropical rain forest. Biotropica 30:214222.

CAVALCANTI, T.B. 1990. Flora da Serra do Cipó, Minas Gerais: Caracterização e lista de espécies. Boletim de Botânica da Universidade de São Paulo 12:67-93.

CHAMBERS, J.C. 1993. Seed and vegetation dynamics on an alpine herb field - effects of disturbance type. Canadian Journal of Botany 71:471-485.

CLARKE, P.J. \& KNOX, K.J.E. 2002. Post-fire response of shrubs in the tablelands of eastern Australia: do existing models explain habitat difference? Australian Journal of Botany 50:53-62.

CLARKE, P.J., KNOX, K.J.E., WILLS, K.E. \& CAMPBELL, M. 2005. Landscape patterns of woody plant response to crown fire: disturbance and productivity influence sprouting ability. Journal of Ecology 93:544-555.

CORDEIRO, I. 1992. Flora da Serra do Cipó, Minas Gerais: Euphorbiaceae. Boletim de Botânica da Universidade de São Paulo 13:169-217.

DUPUY, J.M. \& CHAZDON, R.L. 1998. Long-term effects of forest regrowth and selective logging on the seed bank of tropical forests in NE Costa Rica. Biotropica 11:223-237.

ELLISON, A.M., DENSLOW, A.M., LOISELLE, B.A. \& BRENES, D.M. 1993. Seed and seedling ecology of neotropical Melastomataceae. Ecology 74:1737-1749.
ERSCHBAMER, B., KNERINGER, E. \& SCHLAG, R.N. 2001. Seed rain, soil seed bank, seedling recruitment and survival of seedlings on a glacier foreland in the Central Alps. Flora 196:304-312.

FENNER, M. 1995. Ecology of seed banks. In Seed development and germination (J. Kiegel \& G. Galili, eds.). Marcel Dekker, New York, p.507-528

FONSECA, C.E.L. \& WALTER, B.M.T. 2001. As matas de galeria no contexto do bioma Cerrado. In Cerrado caracterização e recuperação de matas de galeria (J.F. Ribeiro, C.E.L. Fonseca \& J.C. Souza, eds.). Embrapa, Planaltina, p.29-47.

GALLAGHER, R.S. \& CARDINA, J. 1997. Soil water thresholds for photoinduction of redroot pigweed germination. Weed Science 45:414-418.

GALVÃO, M.V. \& NIMER, E. 1965. Clima. In Geografia do Brasil - Grande Região Leste, v.5. Instituto Brasileiro de Geografia e Estatística, Rio de Janeiro, p.91-139.

GARCIA, Q.S. \& DINIZ, I.S.S. 2003. Comportamento germinativo de três espécies de Vellozia da Serra do Cipó (MG). Acta Botanica Brasilica 17:487-494.

GARCIA, Q.S., JACOBI, C.M. \& RIBEIRO, B.A. 2007. Resposta germinativa de duas espécies de Vellozia (Velloziaceae) dos campos rupestres de Minas Gerais, Brasil. Acta Botanica Brasilica 21:451-456.

GARWOOD, N.C. 1989. Tropical soil seed banks: a review. In Ecology of soil seed banks (M.A. Leck, V.T. Parker \& R.L Simpson, eds.). Academic Press, San Diego, p. 149-209

GERMAINE, H.L. \& MCPHERSON, G.R. 1999. Effects of biotic factors on emergence and survival of Quercus emoryi at lower treeline, Arizona, USA. Ecoscience 6:92-99.

GIULIETTI, A.M. \& MENEZES, N.A. 2000. Campo Rupestre. In Lista vermelha das espécies ameaçadas de extinção de Minas Gerais (M.P. Mendonça \& L.V. Lins, eds.). Fundação Biodiversitas/Fundação Zoobotânica, Belo Horizonte, p.76-83

GIULIETTI, A.M., MENEZES, N.A., PIRANI, J.R. \& WANDERLEY, M.G.L. 1987. Flora da Serra do Cipó: caracterização e lista de espécie. Boletim de Botânica da Universidade de São Paulo 9:1-151.

GOMES, V., MADEIRA, J.A., FERNANDES, G.W. \& LEMOS FILHO, J.P. 2001. Seed dormancy and germination of sympatric species of Chamaecrista (Leguminosae) in a rupestrian field. International Journal of Ecology and Environmental Sciences 27:191-197.

GUARDIA, R., GALLART, F. \& NINOT, J.M. 2000. Soil seed bank and seedling dynamics in badlands of the Upper Llobregat basin (Pyrenees). Catena 40:189-202.

GUNSTER, A. 1994. Seed bank dynamics - longevity, viability and predation of seeds of serotinous plants in the central Namib desert. Journal of Arid Environments 28:195-205.

HOLL, K.D. 1999. Factors limiting rain forest regeneration in abandoned pasture: seed rain, seed germination, microclimate and soil. Biotropica 31:229-242. 
HOLMES, P.M. \& COWLING, R.M. 1997. Diversity, composition and guild structure relationships between soil-stored seed banks and mature vegetation in alien plant-invaded South African fynbos shrublands. Plant Ecology 133:107-122

HOPKINS, M.S., TRACEY, J.G. \& GRAHAM, A.W. 1990. The size and composition of soil seed-banks in remnant pathes of tree structural rainforest types in North Queensland. Australian Journal of Ecology 15:43-50.

HOULE, G. \& PHILLIPS, D.L. 1988. The soil seed bank of granite outcrop plant communities. Oikos 52:87-93.

JENSEN, K. 1998. Species composition of soil seed bank and seed rain of abandoned wet meadows and their relation to above ground vegetation. Flora 193:345-359.

JORGENSEN, P. M. \& ULLOA, U. 1994. Seed plants of the high Andes of Equador - a check list. AAU Reports 34:45-70.

KIRKMAN, L.K. \& SHARITZ, R.R. 1994. Vegetation disturbance and maintenance of diversity in intermittently flooded Carolina Bays, in South Carolina. Ecological Applications 4:177-188.

LECK, M.A. 1989. Wetland seed banks. In Ecology of soil seed banks (M.A. Leck, V.T. Parker \& R.L Simpson, eds.). Academic Press, California, p.123-147

LECK, M.A. \& BROCK, M.A. 2000. Ecological and evolutionary trends in wetlands: evidence from seeds and seed banks in South Wales, Australia and New Jersey, USA. Plant Species Biology 15:97-112.

LORENZI, H. 2000. Plantas Daninhas do Brasil. 3a ed. Plantarum, Nova Odessa.

LOUDA, S.M. 1989. Predation in the dynamics of seed regeneration. In Ecology of soil seed banks (M.A. Leck, V.T. Parker \& R.L. Simpson, eds). Academic Press, San Diego, p.25-51

MADEIRA, J.A. \& FERNANDES, G.W. 1999. Reproductive phenology of sympatric taxa of Chamaecrista (Leguminosae) in Serra do Cipó, Brazil. Journal of Tropical Ecology 15:463-479.

MAGURRAN, A.E. 1988. Ecological diversity and its measurement. Cambridge University Press, Cambridge.

MARQUES, M.C.M. 1998. Polígalas do Brasil V. Seção Polygala (Polygalaceae). Arquivos do Jardim Botânico do Rio de Janeiro 29:1-114

MASAKI, T., TANAKA, H., SHIBATA, M. \& NAKASHIZUKA, T. 1998. The seed bank dynamics of Cornus controversa and their role in regeneration. Seed Science Research 8:53-63.

MEGURO, M., PIRANI, J.R., MELLO-SILVA, R. \& GIULIETTI, A.M. 1996. Caracterização florística e estrutural de matas ripárias e capões de altitude da Serra do Cipó, Minas Gerais. Boletim de Botânica da Universidade de São Paulo 15:13-29.

MOLES, A.T. \& DRAKE, D.R. 1999. Potential contributions of the seed rain and the seed bank to regenerate forest under plantation pine in New Zealand. New Zealand Journal of Botany 37:83-93.
OLIVEIRA, P.G. \& GARCIA, Q.S. 2005. Efeitos da luz e da temperatura na germinação de sementes de Syngonanthus elegantulus, S. elegans e S. venustus (Eriocaulaceae). Acta Botanica Brasilica 19:627-633.

PARKER, V.T., SIMPSON, R.L. \& LECK, M.A. 1989. Pattern and process in the dynamics of seed banks. In Ecology of soil seed banks (M.A. Leck, V.T. Parker \& R.L Simpson, eds.). Academic Press, California, p.367-384

PEARSON, T.R.H., BURSLEM, D.F.R.P., MULLINS, C.E. \& DALLING, J.W. 2002. Germination ecology of neotropical pioneers: interacting effects of environmental conditions and seed size. Ecology 83:2798-2807.

PIERCE, S.M. \& COWLING, R.M. 1991. Dynamics of soilstored seed banks 6 shrubs in fire-prone Fynbos. Journal of Ecology 79:731-747.

RANIERI, B.D., LANA, T.C., NEGREIROS, D., ARAÚJO, L.M. \& FERNANDES, G.W. 2003. Germinação de sementes de Lavoisiera cordata e Lavoisiera francavillana (Melastomataceae), espécies simpátricas da Serra do Cipó, Brasil. Acta Botanica Brasilica 17:523-530.

RIBEIRO, K.T. \& FERNANDES, G.W. 2000. Pattern of abundance of a narrow endemic species in a tropical and infertile montane habitat. Plant Ecology 147:205-218.

ROBINSON, H. 1999. Generic and subtribal classification of American Vernonieae. Smithsonian Contribution to Botany 89:1-116.

RODRIGUES, L.A., CARVALHO, D.A., GOMES, L.J. \& BOTREL, R.T. 2002. Espécies vegetais nativas usadas pela população local em Luminárias, MG. Boletim Agropecuário 52:1-34.

ROMERO, R. \& MARTINS, A.B. 2002. Melastomataceae from Serra da Canastra National Park, Minas Gerais, Brasil. Revista Brasileira de Botânica 25:19-24.

SASSAKI, R.M., RONDON, J.N., ZAIDAN, L.B.P. \& FELIPPE, G.M. 1999. Number of buried seeds and seedlings emergence in cerradão, cerrado and gallery forest soils at Pedregulho, Itirapina (SP), Brazil. Revista Brasileira de Botânica 22:147-152.

SETTERFIELD, S.A. 2002. Seedling establishment in an Australian tropical savanna: effects of seed supply, soil disturbance and fire. Journal of Applied Ecology 39: 949-959.

SHEPHERD, G.J. 1994. FITOPAC 1: manual de usuário. Departamento de Botânica, Universidade Estadual de Campinas, Campinas.

SILVA JÚNIOR, M.C., FELFILI, J.M., WALTER, B.M.T., NOGUEIRA, P.E., REZENDE, A.V., MORAIS, R.O. \& NÓBREGA, M.G.G. 2001. Análise da flora arbórea de matas de galeria no Distrito Federal: 21 levantamentos. In Cerrado - caracterização e recuperação de matas de galeria. (J.F. Ribeiro, C.E.L. Fonseca \& J.C. Souza Silva, eds.). Embrapa, Planaltina, p.143-191.

SILVEIRA, F.A.O., NEGREIROS, D. \& FERNANDES, G. W. 2004. Germinação de sementes de Marcetia taxifolia (St. Hil.) DC. (Melastomataceae): influência da luz e da temperatura. Acta Botanica Brasilica 18:847-851. 
SILVEIRA, F.A.O., FERNANDES, F. \& FERNANDES, G.W. 2005. Light and temperature influence on seed germination of Calliandra fasciculata Benth. (Leguminosae). Lundiana 6:95-97

SOKAL, R.R. \& ROHLF, F.J. 1995. Biometry. $3^{\text {rd }}$ ed. W.H. Freeman, New York.

SOUSA-SILVA, J.L., RIBEIRO, J.F., FONSECA, C.E.L. \& ANTUNES, N.B. 2001. Germinação de sementes e emergência de plântulas de espécies arbóreas e arbustivas que ocorrem em matas de galeria. In Cerrado - caracteização e recuperação de matas de galeria. (J.F. Ribeiro, C.E.L. Fonseca \& J.C. Souza-Silva, orgs.). Embrapa, Planaltina, p.379-422.

SOUZA, H.C. 1997. Estudo comparativo de adaptações anatômicas em órgãos vegetativos de espécies de Lavoisiera DC. (Melastomataceae) da Serra do Cipó, MG. Dissertação de mestrado, Universidade de São Paulo, São Paulo.

THOMPSON, K. \& GRIME, J.P. 1979. Seasonal variation in the seed banks of herbaceous species in ten contrasting habitats. Journal of Ecology 67:893-921.

VAN DER VALK, A.G. \& PEDERSON, R.L. 1989. Seed banks and the management and restoration of natural vegetation. In Ecology of soil seed banks (M.A. Leck, V.T. Parker \& R.L Simpson, eds.). Academic Press, California, p.329-346.

VAZQUEZ-YANES, C.V. \& OROZCO-SEGOVIA, A. 1993. Patterns of seed longevity and germination in the tropical rainforest. Annual Review of Ecology and Systematics 24:69-87.
VEENENDAAL, E.M., ERTNST, W.H.O. \& MODISE, G.S. 1996. Effect os seasonal rainfall pattern on seedling emergence and establishment of grasses in a savanna in south-eastern Botswana. Journal of Arid Environments 32:305-317.

VELTEN, S.B. \& GARCIA, Q.S. 2005. Efeitos da luz e da temperatura na germinação de Eremanthus (Asteraceae), ocorrentes na Serra do Cipó, MG, Brasil. Acta Botanica Brasilica 19:755-763.

VIANA, L.R., FERNANDES, G.W. \& SILVA, C.A. 2005. Ecological road threatens endemic Brazilian plant with extinction. Plant Talk 41:15.

WILLEMS, J.H. 1995. Soil seed bank, seedling recruitment and actual species composition in an old and isolated chalk grassland site. Folia Geobotanica et Phytotaxonomica 30:141-156.

WILLIAMS LINERA, G. 1993. Soil seed banks in four lower montane forest of Mexico. Journal of Tropical Ecology 9:321-337.

YATES, C.J. \& LADD, P.G. 2004. Breeding system, pollination and demography in the rare granite endemic shrub Verticordia staminosa ssp. Staminosa in south-west Western Australia. Austral Ecology 29:189-200

YU, S.L., STERNBERG, M., JIANG, G.M. \& KUTIEL, P. 2003. Heterogeneity in soil seed banks in a Mediterranean coastal sand dune. Acta Botanica Sinica 45:536-543.

ZAPPI, D.C. 1994. Pilosocereus (Cactaceae). The genus in Brazil. Succulent Plant Research 3:1-160. 